\title{
Adaptation de la mise en œuvre des bonnes pratiques cliniques en fonction des caractéristiques de certaines recherches
}

\author{
Pierre-Henri Bertoye, ${ }^{1}$ Soizic Courcier-Duplantier ${ }^{2}$, Nicolas Best ${ }^{3}$ \\ et les participants à la table ronde $n^{\circ} 2$ de Giens XXI* \\ 1 Afssaps, Saint Denis, France \\ 2 Bristol Myers Squibb, Rueil Malmaison, France \\ 3 DRRC, Hôpital Saint Louis, Paris, France
}

\begin{abstract}
Mots clés :
essais cliniques;

$\mathrm{BPC}$;

adaptation ;

mise en œuvre ;

risques ;

enjeux ;

monitoring;

circuit du médicament ; pharmacovigilance

Résumé - La réalisation des essais cliniques se fait dans un cadre réglementaire strict. L'objectif de cette table ronde était d'élaborer des recommandations raisonnables pour la mise en œuvre des bonnes pratiques cliniques (BPCs) dans certains types de recherches, compte-tenu de leurs caractéristiques et enjeux. Deux types de risque à prendre en compte ont été identifiés : ceux liés aux caractéristiques de la recherche et ceux liés à l'impact des résultats de la recherche. Une grille d'évaluation de ces risques a été élaborée. Les travaux de la table ronde se sont articulés autour de 3 thématiques principales : l'adaptation du monitoring, le médicament expérimental, les effets indésirables. Trois méthodes d'adaptation du monitoring ont été passées en revue avec liste des avantages et inconvénients : l'approche graduelle, le monitoring centralisé, le monitoring par sondage. La réflexion sur le médicament expérimental s'est organisée autour du circuit du (des) médicament(s). L'arbre décisionnel «de base » suivant est recommandé : 1) le médicament est-il expérimental ? 2) la conception et les objectifs de l'essai nécessitent ils un conditionnement spécifique à la recherche? 3) le risque d'utilisation est-il supérieur à celui de la pratique courante?

Enfin, il est apparu que l'adaptation de la mise en œuvre des BPCs en terme de pharmacovigilance était très limitée et qu'elle pouvait éventuellement se concevoir pour le médicament, objet de la recherche, qui a déjà une autorisation de mise sur le marché (AMM) et dont le profil de sécurité est bien connu; dans ce cas, seule une simplification du recueil des évènements indésirables non graves est envisageable, qui peut se faire via l'élaboration et l'utilisation de listing standardisé de recueil. L'adaptation de la mise en œuvre des BPCs est possible. Elle doit tenir compte des caractéristiques de la recherche : quels objectifs/quels risques/quels enjeux. Les choix en terme d'adaptation doivent être prédéfinis, documentés et justifiés; si nécessaire ils seront aussi réévalués en cours d'étude.
\end{abstract}

Un essai clinique est destiné à obtenir des résultats répondant de façon crédible à la question posée sur un médicament ou une stratégie thérapeutique, sans faire courir de risques injustifiés aux personnes qui s'y prêtent. ${ }^{[1]}$ La qualité de la conception, de la mise en œuvre et du suivi d'un essai clinique conditionne d'une part la sécurité et la protection des personnes qui s'y prêtent et d'autre part la fiabilité des résultats obtenus sur un médicament ou une stratégie thérapeutique, que ces résultats fassent l'objet d'une publication et/ou d'un dossier d'autorisation de mise sur le marché (AMM).

\footnotetext{
^ Pour la liste des participants, voir en fin d'article.
}

Ce respect de la qualité s'organise et se contrôle par des procédures systématiquement appliquées, et correspondant à la mise en œuvre des Bonnes Pratiques Cliniques (BPCs). ${ }^{[2]}$

Le cadre règlementaire strict pour la réalisation des essais cliniques a récemment été modifié et harmonisé au niveau européen par la directive 2001/20/CE du 4 avril 2001 ${ }^{[3]}$ « concernant le rapprochement des dispositions législatives, règlementaires et administratives des Etats membres relatives à l'application de Bonnes Pratiques Cliniques dans la conduite d'essais cliniques de médicaments à usage humain ».

Cette directive a fait l'objet d'une transposition en France dans la loi de Santé Publique du 9 août 2004 (livre $1^{\text {er }}$, titre II du 
code de la santé publique $)^{[4]}$ et dont le décret d'application devrait être prochainement publié. ${ }^{[5]}$

Une directive d'application de la directive 2001/20/CE et destinée à l'application des BPCs, la directive 2005/28/CE du 8 avril $2005,{ }^{66]}$ appelle dans ses attendus et son premier chapitre un projet de guide relatif à des modalités spécifiques pour la mise en œuvre de ces BPCs pour les essais académiques. Ce projet de guide «specific modalities for academic research » est en cours d'élaboration au niveau européen et devrait être mis en consultation dans le courant de l'année 2006.

Dans ce contexte, l'objectif de la table ronde $n^{\circ} 2$ des Rencontres Nationales de Pharmacologie de Giens XXI, était d'élaborer des recommandations raisonnables pour la mise en œuvre des BPCs dans le cadre de certains types de recherches, tout en tenant compte des enjeux de ces recherches.

Ces recommandations avaient pour but :

- d'une part de prolonger des réflexions ou des pratiques déjà en application dans certaines structures de recherche,

- d'autre part d'établir un point de vue consensuel entre les différents partenaires (institutionnels, cliniciens, patients, industriels) qui puisse être présenté et discuté au niveau communautaire pour enrichir les débats sur le guide européen «specific modalities for academic research » en cours d'élaboration (France et Grande-Bretagne en étant les pays rapporteurs).

Le périmètre de la réflexion fut tout d'abord défini de façon précise, consistant en la définition du type de recherches pour lesquelles l'adaptation de la mise en œuvre des BPCs était envisageable.

De façon consensuelle, il fut décidé que les travaux de la table ronde :

- ne concerneraient que les recherches portant sur des médicaments, bénéficiant obligatoirement de l'AMM,

- excluraient toutes les études post-AMM à visée « règlementaire », c'est-à-dire réalisées de façon prospective soit dans le cadre d'une AMM conditionnelle, soit dans le cadre de la recherche d'une nouvelle indication,

- se limiteraient aux essais cliniques interventionnels, et n'aborderaient pas les études non interventionnelles dont la réglementation française doit fixer le périmètre exact.

Ainsi, à titre d'exemples, ont été retenues dans le champ de la table ronde :

- les études cliniques de sécurité post-AMM telles que décrites dans le guide $\mathrm{ICHE}_{2} \mathrm{E}^{[7]}$ visant à étudier les risques potentiels identifiés -effets indésirables ou évènements indésirables- qui nécessitent une évaluation et/ou une caractérisation plus précise,
- les études cliniques visant à préciser une indication (souspopulation, contre-indication...) menant à des publications pouvant influer sur des recommandations médicales ou thérapeutiques de bonnes pratiques; (exemple : aspirine chez l'insuffisant rénal en prévention des accidents thromboemboliques),

- les études cliniques de comparaison de stratégies thérapeutiques ou diagnostiques.

L'identification et l'évaluation des risques et des enjeux de la recherche est ensuite apparue indispensable, avant d'envisager toute adaptation dans la mise en œuvre des BPCs : ${ }^{[8]}$ identifier pour les patients de l'essai le risque lié au produit, celui lié aux investigations, ainsi que le risque lié à la qualité des résultats de la recherche pour la population cible. Le Medical Research Council, en Grande Bretagne, a travaillé sur une telle analyse de risque, mais avec pour conséquence des algorithmes complexes de définition du niveau de monitoring. ${ }^{[9]}$

Cette évaluation doit être entreprise le plus tôt possible, doit être décrite, documentée et détaillée de façon à mettre en regard de ces risques et de ces enjeux un véritable plan de suivi de la recherche. ${ }^{[1]}$

Deux types de risque ont été identifiés :

- tout d'abord les risques liés aux caractéristiques de la recherche :

○ patients de l'essai (populations à risque particulier?...),

- médicament (toxicité particulière?...),

- dangers liés au protocole (examens invasifs, modifications du plan de soins habituels des malades...),

- conception et schéma de l'essai,

- structure en charge de la recherche (expérience en recherche clinique ?...);

- ensuite les enjeux liés à l'impact des résultats de la recherche - enjeux sur les pratiques thérapeutiques (les résultats vontils avoir un impact et quel risque y a-t-il en cas de résultats de l'essai erronés?),

- enjeux économiques,

- besoins de résultats et difficulté de dupliquer l'essai si nécessaire.

Afin d'évaluer au mieux ces risques et enjeux, le groupe a élaboré une grille d'évaluation (Tableau I) permettant de passer en revue chacun des critères cités précédemment et de l'évaluer, dans le contexte de la recherche, sur une échelle de graduation cotée de 1 (faible risque) à 4 (risque élevé).

Cette échelle doit être appliquée comme un continuum au sein duquel chaque critère devra être pondéré. Il est proposé de tester de façon prospective cette grille sur des recherches académiques et/ou industrielles afin d'évaluer son application, de l'affiner ou de la compléter. 
Tableau I. Gradation du risque, du moins risqué (1) au plus risqué (4).

\begin{tabular}{|c|c|c|c|c|}
\hline Niveau de risque & 1 & 2 & 3 & 4 \\
\hline Population (P) & Indications de l'AMM & & $\begin{array}{l}\text { Ages extrêmes, } \\
\text { femmes enceintes }\end{array}$ & $\begin{array}{l}\text { Défaillance significative } \\
\text { d'un système }\end{array}$ \\
\hline$\overline{\text { Médicament }(P)}$ & $\begin{array}{l}\text { Synthèse des } \\
\text { connaissances rassurante } \\
\text { et stabilisée }\end{array}$ & $\begin{array}{l}\text { Synthèse des connais- } \\
\text { sances rassurante et non } \\
\text { totalement stabilisée }\end{array}$ & & $\begin{array}{l}\text { AMM récente }(<2 \text { ans }) \\
\text { Synthèse des connaissances non } \\
\text { rassurante ou non stabilisée }\end{array}$ \\
\hline $\begin{array}{l}\text { Durée de traitement/ } \\
\text { posologie }(P)\end{array}$ & & & & $\begin{array}{l}\text { Usage inhabituel : dose, durée, } \\
\text { association }\end{array}$ \\
\hline $\begin{array}{l}\text { Autres procédures } \\
\text { du protocole }\end{array}$ & $\begin{array}{l}\text { Peu invasif (prise de sang) } \\
\text { (P) }\end{array}$ & $\begin{array}{l}\text { Sevrage potentiellement } \\
\text { dangereux d'un traitement } \\
(\mathrm{P})\end{array}$ & $\begin{array}{l}\text { Modifications du plan } \\
\text { de soins habituel des } \\
\text { malades }\end{array}$ & $\begin{array}{l}\text { Procédure invasive comportant un } \\
\text { risque vital, procédures } \\
\text { complexes/multiples } \\
(\mathrm{P} / \mathrm{E})\end{array}$ \\
\hline Critère principal $(E)$ & Robuste & & Critères composites & Risque d'erreur important \\
\hline $\begin{array}{l}\text { Conséquences } \\
\text { des résultats : } \\
\text { population cible (E) }\end{array}$ & & & Gravité de la pathologie & Essai qui ne pourra pas être répété \\
\hline $\begin{array}{l}\text { Impact économique } \\
\text { des résultats }(E)\end{array}$ & & & & Coût important pour la collectivité \\
\hline
\end{tabular}

Une fois le périmètre défini, les risques et les enjeux évalués, les travaux de la table ronde se sont articulés autour de 3 thématiques principales :

- le monitoring,

- le médicament expérimental,

- les effets indésirables.

\section{L'adaptation du monitoring}

La réflexion sur l'adaptation du monitoring s'est centrée autour de différentes questions :

\subsection{Quelles sont les contraintes règlementaires et que peuton adapter?}

Le texte des BPCs du consensus ICH, dans son paragraphe $5.18 .3,{ }^{[2]}$ nous rappelle tout d'abord que tout essai doit être monitoré :

«The sponsor should ensure that the trials are adequately monitored. The sponsor should determine the appropriate extent and nature of monitoring. The determination of extent and nature of monitoring should be based on considerations such as the objective, purpose, design, complexity, blinding, size, and endpoints of the trial. In general there is a need for on-site monitoring, before, during and after the trial; however, in exceptional circumstances the sponsor may determine that central monitoring in conjunction with procedures such as investigators' training and meetings, and extensive written guidance can assure appropriate conduct of the trial in accordance with Good Clinical Practices. Statistically controlled sampling may be an acceptable method for selecting the data to be verified $»$.

Dans ce texte, il est donc explicite que la détermination de l'étendue et des modalités du monitoring mis en œuvre doit tenir compte de l'objectif, de la complexité, et du type de recherche. Si, dans la majorité des cas, le contrôle sur site doit être favorisé, dans certains cas et sous certaines conditions le texte des BPC rend possible la mise en oeuvre d'un monitoring centralisé. Un monitoring par sondage est une possibilité que peut également considérer le promoteur.

Le promoteur aura donc à évaluer préalablement le monitoring le plus efficient à mettre en œuvre de manière réaliste, qui peut être une combinaison des modalités présentées ci-dessous. 


\subsection{Sur quels critères décider d'une adaptation du monitoring?}

\subsection{1. $~^{\text {re }}$ méthode : l'approche graduelle}

Cette approche appliquée par la Délégation à la Recherche Clinique (DRC) de l'Assistance Publique-Hôpitaux de Paris (APHP) est un exemple d'adaptation déjà mis en œuvre, tenant compte du niveau de risque lié à la recherche et au produit, et visant à garantir les aspects sécuritaires de la recherche clinique tout en tenant compte des moyens disponibles.

Tous les projets promus par la DRC sont soumis à une analyse basée sur le rapport bénéfice/risque pour les sujets participants.

Quatre classes de risque sont prises en compte:

- risque A : risque prévisible faible ou négligeable (ne concerne pas les essais de médicaments),

- risque $\mathrm{B}$ : risque prévisible proche de celui des soins usuels,

- risque $\mathrm{C}$ : risque prévisible élevé,

- risque $\mathrm{D}$ : risque prévisible très élevé.

Les recherches sont classifiées par niveaux de risque (Tableau II), et un niveau graduel de monitoring est mis en oeuvre en fonction de ce niveau (Tableau III).

Une évaluation prospective de cette approche sera réalisée dans le cadre de l'étude OPTIMON OPTImisation du MONitorage des études de recherche clinique institutionnelle, coordonnée par Geneviève Chêne (URC du CHU de Bordeaux \& INSERM U593), Philippe Ravaud (URC de l'Hôpital Bichat) et Jean-Pierre Pignon (IGR Villejuif). Il s'agit d'une étude comparative, randomisée (unité $=$ centre clinque), sans insu, multicentrique, visant à comparer deux stratégies de monitorage : monitoring classique et monitoring selon une approche graduelle inspirée de celle décrite ci-dessus. L'étude devrait débuter au dernier trimestre 2006.

Cette étude intégrera l'approche graduelle présentée dans le tableau I, qui ajoute aux critères de risque liés aux produits expérimentaux et aux investigations de la recherche, des considérations relatives à la population étudiée, à la complexité de la conception de l'essai et à l'impact de la qualité des résultats. C'est pourquoi il a été convenu que les critères pris en compte pour cette approche graduelle, qui a été formalisée par la DRC de l'AP-HP il y a quelques années en vue de réorganiser la gestion des essais pour lesquels elle était le promoteur, seraient complétés par d'autres éléments listés dans le tableau I.

\subsection{2. $2^{e}$ méthode : le monitoring centralisé}

Le monitoring centralisé est défini par l'organisation systématique de la remontée de données prédéfinies essentielles à l'essai vers le moniteur central. La mise en oeuvre d'un monitoring centralisé ne peut s'envisager, de l'avis du groupe, sans un minimum de monitoring sur site. ${ }^{[2]}$

Les avantages du monitoring centralisé sont :

- la possibilité d'espacer le rythme de visites sur site avec, de ce fait, une économie des coûts liés à ces visites,

- une plus grande souplesse dans la gestion de l'essai,

- une meilleure efficacité dans la préparation du monitoring sur site,

- la concentration en un lieu des compétences sur le produit et la maladie.

En revanche, il faut être conscient des contraintes :

- une distance par rapport à la réalité du centre,

- une vérification du respect des obligations réglementaires moins fréquente et détection plus tardive des non-conformités,

- une remontée d'informations dépendante de la compréhension du protocole par les investigateurs et nécessitant des actions importantes et répétées de formation et information des investigateurs,

- le déplacement de la charge de travail sur l'investigateur,

- des contraintes liées à la remontée d'informations confidentielles (ex : copies de comptes rendus de biologie devant être rendues anonymes).

Enfin cette méthode est facilitée par l'utilisation de cahiers électroniques, qui toutefois impose d'autres contraintes pour garantir l'origine des données (signature électronique) et leur intégrité (piste d'audit sécurisée).

\subsection{3. $3^{e}$ méthode : le monitoring par sondage}

Trois niveaux d'échantillonnage sont possibles :

- échantillonnage des données,

- échantillonnage des observations,

- échantillonnage des centres.

Dans tous les cas, lorsque cette technique est mise en œuvre, elle doit s'accompagner d'une stratégie prédéfinie d'actions à mettre en oeuvre en fonction du résultat observé, c'est-à-dire qu'il faut non seulement vérifier la qualité de l'échantillon mais surtout définir une attitude en fonction de la qualité qui sera observée.

Ainsi pourront être mises en œuvre des règles, préalablement définies et adaptées au cas par cas, soit à la hausse (pour tous les centres) lorsque la qualité de l'échantillon est insuffisante, soit à la baisse (pour le centre considéré) lorsque la qualité de l'échantillon est jugée satisfaisante.

Il faut toutefois souligner que dans un essai clinique les variables de mesure sont d'importance inégale pour les résultats de 
Tableau II. Classification des recherches par niveau de risque-DRC AP-HP.

\begin{tabular}{|c|c|c|c|c|c|}
\hline $\begin{array}{l}\text { Niveaux } \\
\text { de risque }\end{array}$ & $\begin{array}{l}\text { Essai clinique } \\
\text { Thérapie génique } \\
\text { ou cellulaire }\end{array}$ & $\begin{array}{l}\text { Physiopathologie } \\
\text { Génétique }\end{array}$ & $\begin{array}{l}\text { Questionnaires } \\
\text { Qualité de vie } \\
\text { Psychiatrie }\end{array}$ & $\begin{array}{l}\text { Imagerie } \\
\text { Radiologie } \\
\text { Radiothérapie } \\
\text { Isotopes Chirurgie }\end{array}$ & Dispositifs Médicaux \\
\hline Risque B & $\begin{array}{l}\text { Phase IV } \\
\text { Phase III } \\
\text { d'association } \\
\text { de produits avec AMM }\end{array}$ & $\begin{array}{l}\text { Invasif selon type d'acte } \\
\text { avec injection de produit } \\
\text { selon produit }\end{array}$ & $\begin{array}{l}\text { Si questionnaire } \\
\text { pointu dans } \\
\text { pathologie grave }\end{array}$ & $\begin{array}{l}\text { Technique Standard } \\
\text { mais mal connue } \\
\text { Chirurgie en routine }\end{array}$ & $\begin{array}{l}\text { DM marqués CE en Ila, Ilb ou } \\
\text { III en routine DM CE en Ila } \\
\text { hors indication DM non CE en } \\
\text { I (sauf invasif ou actif) }\end{array}$ \\
\hline Risque C & $\begin{array}{l}\text { Phase III } \\
\text { Nouvelle indication } \\
\text { Population à risque }\end{array}$ & $\begin{array}{l}\text { Invasif selon type d'acte } \\
\text { avec injection. de produit } \\
\text { selon produit }\end{array}$ & - & $\begin{array}{l}\text { Phase d'apprentissage } \\
\text { Généralisation } \\
\text { d'une nouvelle } \\
\text { technique }\end{array}$ & $\begin{array}{l}\text { DM marqués CE en Ilb hors } \\
\text { indication DM marqués CE en } \\
\text { III avec peu de recul }\end{array}$ \\
\hline Risque D & Phase I ou II & - & - & $\begin{array}{l}\text { Nouvelle technique } \\
\text { Mise au point }\end{array}$ & $\begin{array}{l}\text { DM marqués CE en III hors } \\
\text { indication DM non CE en I } \\
\text { (invasif ou actif) Ila, Ilb ou III }\end{array}$ \\
\hline
\end{tabular}

Tableau III. Niveau de monitoring en fonction du risque-DRC AP-HP.

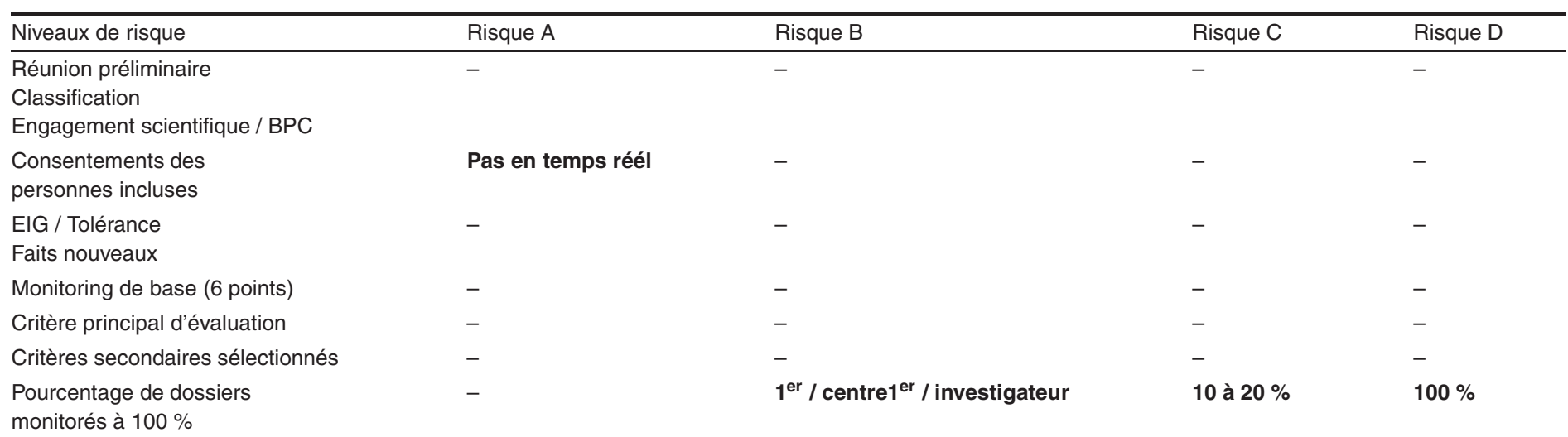

BPC : bonnes pratiques clinique; EIG : événement indésirable grave.

l'essai, et donc les erreurs notées ont des conséquences inégales en termes de gravité; le nombre d'erreurs acceptables doit donc tenir compte de cette gravité. D'autre part certaines erreurs, liées à des évènements rares sont difficiles à détecter.

\section{L'adaptation des mesures relatives au médicament expérimental}

La réflexion sur le médicament expérimental s'est organisée autour du circuit du (des) médicament(s), de la traçabilité (étiquetage, dispensation, observance).

Cette réflexion a été menée au travers d'exemples concrets et pour chaque exemple ont été analysés les enjeux en terme de risque, d'impact sur la qualité des résultats et d'impact sur les pratiques médicales avec à la clé des propositions d'allègement pour certains cas.

En préalable à ce travail, il est apparu au groupe qu'il était avant tout nécessaire de définir clairement si la recherche est bien un essai de médicament, et si tel est le cas, de définir clairement quel est le médicament expérimental. La définition du médicament expérimental («investigational medicinal product») dans la directive 2001/20/CE ${ }^{[3]}$ est la suivante : « a pharmaceutical form of an active substance or placebo being tested or used as a reference in a clinical trial, including products already with a marketing authorisation but used or assembled (formulated or packaged) in a way different from the authorised form, or when used for an unauthorised indication, or when used to gain further information about the authorised form ». 
Cette définition fait actuellement l'objet de discussions au niveau communautaire ainsi que de clarifications sur les exigences requises en matière de circuit et traçabilité, selon les différents scenarios d'essais possibles.

La question, notamment dans les essais comparatifs, des traitements standards de prise en charge de la pathologie (background therapy) est cruciale : la recommandation du groupe est d'essayer au maximum de sortir le(s) traitement(s) standard(s) de la définition du médicament expérimental si l'objectif de l'essai ne pose aucune question spécifique et directe sur ce traitement de fond, et si, bien sûr, l'utilisation de ce traitement standard est validée par l'AMM.

Les textes de $\mathrm{BPCs}^{[6]}$ et de Bonnes Pratiques de fabrication ${ }^{[10]}$ prévoient que les médicaments expérimentaux font l'objet d'un étiquetage spécifique ainsi que d'une documentation de leur circuit et de leur comptabilité (traçabilité).

L'organisation du mode de traçabilité doit avant tout répondre aux questions suivantes : l'absence éventuelle d'étiquetage spécifique :

- A-t-il un impact sur la sécurité des patients ?

- A-t-il un impact sur les résultats de l'essai?

- A-t-il un impact sur la mesure de l'observance?

À partir de différents exemples qui ont été passés en revue, le groupe a élaboré un arbre décisionnel de «base » simple. Il tient compte de trois éléments :

- facteur 1 : le médicament est-il expérimental ? Est-il donc bien inclus dans la définition de l'IMP (Investigational Medicinal Product) dans le cadre de l'étude?

- facteur 2 : la conception et les objectifs de l'essai nécessitentils un conditionnement spécifique à la recherche?

- facteur 3 : le risque d'utilisation ou de détournement est-il supérieur à celui de la pratique courante?

Si les facteurs 1 et 2 au moins sont présents, ou si les facteurs 1 et 3 au moins sont présents, alors un étiquetage spécifique pour la recherche est demandé et indispensable.

Pour les autres cas, des aménagements vis à vis du conditionnement et de l'étiquetage spécifiques pour l'essai peuvent être envisagés au cas par cas en fonction des contraintes liées à l'étude.

Ainsi certaines situations rendent, à l'évidence, difficile mais pas toujours impossible - l'étiquetage et doivent aussi être prises en compte dans l'arbre de décision.

Par exemple :

- lorsque les médicaments expérimentaux ne sont pas clairement définis et fixés par la recherche, mais par exemple seulement identifiés par la classe ATC (Anatomic Therapeutic Chemical),
- ou bien, lorsque le circuit du médicament doit se faire en ambulatoire via des officines de ville.

Enfin, dans d'autres situations, l'étiquetage peut même être considéré comme peu utile :

- lorsque le médicament entre dans une préparation réalisée dans une pharmacie hospitalière (PUI) : seule la préparation destinée à l'essai est étiquetée,

- lorsqu'un lot de médicament identifiable peut être réservé à l'essai clinique,

- lorsque le médicament est administré sous contrôle direct de l'investigateur (administration IV [intra veineuse]) et que la documentation est réalisée en temps réel.

Quelle que soit la solution retenue, il est indispensable et absolument nécessaire, dans tous les cas, et plus encore lorsqu'il y a simplification, qu'une documentation détaillée et adaptée soit établie au préalable et en cours d'étude ainsi qu'un bilan des circuits.

\section{L'adaptation des procédures de gestion/déclaration des évènements indésirables}

Les exigences en terme de vigilance dans le cadre des essais cliniques ont été clairement définies par la directive 2001/20/CE ${ }^{[3]}$ ainsi que dans sa transposition en France au travers de la loi de santé publique $\mathrm{n}^{\circ}$ 2004-806 du 9 août 2004. [ ${ }^{[1]}$

Le recueil et la circulation de l'information concernant les effets indésirables sont particulièrement importants en raison des décisions souvent graves qui peuvent en découler pour l'utilisation du médicament étudié. ${ }^{[1]}$

La réflexion sur l'éventuelle simplification du suivi de la vigilance dans une recherche biomédicale s'est centrée d'abord sur le type d'opérations pouvant être simplifiées (Effet Indésirable Grave Inattendu [«SUSAR »], codage, effets indésirables non graves, déclarations, rapport annuel de sécurité...)

Il est apparu que l'adaptation de la mise en œuvre des BPCs en cette matière était très limitée et qu'elle pouvait éventuellement et uniquement se concevoir pour le médicament, objet de la recherche, qui a déjà une AMM et dont le profil de sécurité est bien connu; dans ce cas et seulement dans ce cas, parmi toutes les exigences requises en matière de vigilance, seule une simplification du recueil des évènements indésirables non graves par l'investigateur est envisageable; ce recueil peut se faire via l'élaboration et l'utilisation de liste standardisée de recueil.

Il est apparu qu'en matière de vigilance des essais cliniques, les actions les plus utiles à envisager étaient une aide aux investigateurs et aux promoteurs. Cette aide doit porter notamment sur 
l'évaluation de la causalité au travers d'une approche systématique prenant en compte la chronologie par rapport à la prise du médicament et la sémiologie de l'évènement. De la même manière une aide/formation des investigateurs pour l'évaluation de la gravité d'un évènement indésirable peut être apportée. Ainsi, au sein de l'ANRS (Agence Nationale de Recherche sur le Sida), a été développée une grille de cotation de la gravité des évènements indésirables. ${ }^{[11]}$

La directive européenne 2001/20/CE du 4 avril 2001, et sa transposition en France dans la loi de Santé Publique du 9 août 2004, renforcent de façon importante la responsabilité du promoteur en matière de vigilance. Face à cela, il apparaît nécessaire pour les recherches institutionnelles de mutualiser les moyens et outils nécessaires à mettre en œuvre pour répondre aux nouvelles exigences. La Circulaire $n^{\circ}$ DHOS/OPRC/2005/252 du 26 mai 2005 se place dans le contexte de la nouvelle gouvernance des Centres Hospitaliers Universitaires (CHU) et définit la nécessité de professionnaliser la recherche clinique et de reconnaître les personnels de recherche par la constitution de départements ou de fédérations de recherche clinique au sein des établissements. D'autre part elle stipule, par appel à projets, la création de structures interrégionales de recherche clinique chargées d'assurer des missions spécifiques d'animation et de soutien de l'activité de recherche, ne pouvant être assurées efficacement au niveau local, notamment, l'appui à la réalisation de certaines missions spécifiques du promoteur telles que système d'assurance qualité, vigilance...

Cette mutualisation pour la vigilance pourrait en particulier concerner :

- les ressources humaines : les «vigilants»,

- les outils informatiques : base de données,

- le référentiel MedDRA pour le codage (licence, formation),

- la formation à Eudravigilance CT module.

En conclusion, l'adaptation de la mise en œuvre des BPCs est possible, et prévue par les textes. Elle doit tenir compte en premier lieu des caractéristiques de la recherche : quels objectifs/quels risques/quels enjeux, plutôt que du type de promoteur (académique ou industriel). Les choix en terme d'adaptation doivent être prédéfinis, documentés et justifiés; si nécessaire ils seront aussi réévalués en cours d'étude. Enfin il existe peu d'allègement possible sur la vigilance.

\section{Participants}

Abiteboul M. (Quintiles), Augiers de Cremiers F. (Wyeth Lederle), Bélorgey C. (Afssaps), Blazejewki S. (Université Victor
Segalen, Bordeaux), Bouxin-Métro A. (ANRS), Chene G. (INSERM, Bordeaux), Couderc M. (Sanofi Synthélabo), DananDurieux M. (Sanofi Aventis), Deneulin A. (Astra Zeneca), Faurisson F. (Eurordis), Libersa C. (CHRU, Lille), Malchiodi M. (Roche), Marquet P. (Hôpital Dupuytren, Limoges), Orefice C. (3M Santé), Postaire E. (DRCT, INSERM, Paris), Rancinan C. (INSERM, Bordeaux), Reynier J.-C. (AP-HM, Marseille), Spriet A. (Spriet Conseil, Paris), Tibi A. (AGEPS, Paris), Toneatti C. (Institut Pasteur, Paris), Treluyer J.-M. (Hôpital Saint Vincent de Paul, Paris), Vicaut A. (Hôpital Fernand Widal, Paris), VincentPelletier A. (CPCET, Marseille).

\section{Références}

1. Bonnes pratiques des Essais Cliniques des médicaments- Alain Spriet (Paris), Thérèse Dupin-Spriet (Lille). Troisième édition. ISBN 3-8055-7723-0. Editions Karger, Paris

2. Note for guidance on Good Clinical practice : ICH harmonised tripartite guidelines, CHMP/ICH/135/95. http: //www . ich.org

3. Directive 2001/20/CE du parlement européen et du conseil du 4 avril 2001 concernant le rapprochement des dispositions législatives, règlementaires et administratives des Etats membres relatives à l'application des bonnes pratiques cliniques dans la conduite d'essais cliniques de médicaments à usage humain. Journal Officiel des Communautés Européennes /1.5.2001 /L121/34 http: //pharmacos. eudra.org

4. Loi $\mathrm{n}^{\circ}$ 2004-806 du 9 août 2004 relative à la politique de santé publique http: //www. legifrance.gouv

5. Décret $n^{\circ}$ 2006-477 du 26 avril 2006 modifiant le chapitre Ier du titre II du livre Ier de la première partie du code de la Santé Publique relatifs aux recherches biomédiacles (dispositions réglementaires) J. O. n 99 du 27 avril 2006 page 6332 texte $\mathrm{n}^{\circ} 10$ http: //www. legifrance.gouv

6. Directive 2005/28/CE de la Commission du 8 avril 2005 fixant des principes et des lignes directrices détaillées relatifs à l'application de bonnes pratiques cliniques en ce qui concerne les médicaments expérimentaux à usage humain, ainsi que les exigences pour l'octroi de l'autorisation de fabriquer ou d'importer ces médicaments. Journal Officiel des Communautés Européennes 9.4.2005/L 91/13

7. ICH Harmonised Tripartite Guideline. Pharmacovigilance planning E2E recommanded for adoption at step 4 of the ICH Process on 18 November 2004 by the ICH Steering Committee http://www.ich.org

8. Essais cliniques, théorie, pratique et critique- G.Bouvenot, M.Vray Editions Flammarion Medecine-Sciences, Paris

9. Medical Research Council, UK, Notes on Good practice for Research Organisations in the Management of a Portfolio of Trials 3: management of risk

10. Directive 2003/94/CE de la Commission du 8 octobre 2003 établissant les principes et lignes directrices de bonnes pratiques de fabrication concernant les médicaments à usage humain et les médicaments expérimentaux à usage humain. Journal Officiel des Communautés Européennes /14.10.2003 /262/22 http: //pharmacos. eudra.org

11. Grille de cotation de gravité des évènements indésirables, disponible sur le site de l'ANRS http://www/anrs. fr

Correspondance et offprints : Soizic Courcier, Bristol Myers Squibb, 3 rue Joseph Monnier, 92578 Rueil Malmaison, France.

E-mail : soizic.courcier@bms.com 